\title{
HEXAVALENT VACCINE IN EUROPE: SAFETY DATA FROM THE RANDOMIZED CLINICAL TRIALS
}

\author{
NOÉMI-BEÁTRIX BULIK ${ }^{1}{ }^{*}$, CAMELIA BUCȘA $^{2}$, ANDREEA FARCAȘ $^{2}$, OVIDIU ONIGA $^{1}$

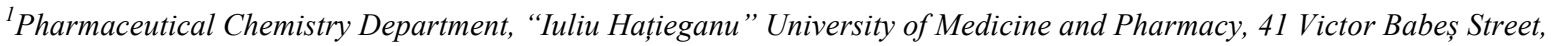 \\ 400012, Cluj-Napoca, Romania \\ ${ }^{2}$ Drug Information Research Centre, "Iuliu Haţieganu” University of Medicine and Pharmacy, 6 Pasteur Street, 400349, \\ Cluj-Napoca, Romania
}

*corresponding author: bulik.noemi@umfcluj.ro

Manuscript received: March 2018

\begin{abstract}
Any vaccine safety issue affects the public confidence in vaccines. This study summarized the safety data published in randomized clinical trials from Europe on the hexavalent vaccine (diphtheria-tetanus-pertussis toxoid, inactivated poliomyelitis virus, antigens against hepatitis B and Haemophilus influenzae type B polysaccharide) administered alone or together with other paediatric vaccines, published between 2000-2016. The solicited adverse events (AEs), unsolicited AEs (uAEs) and serious AEs (SAEs) were evaluated. We included 15 studies summing up 6,618 children with the age between 2 24 months. Grade 3 local reactions ranged between $1.1 \%-15.5 \%$ for redness, $0.4 \%-7.4 \%$ for pain and $1.4 \%-9.7 \%$ for swelling. Irritability and fever were the most frequently reported general symptoms. The incidence of fever ranged between $11.0 \%-57.3 \%$, while grade 3 fever $\left(>39.5^{\circ} \mathrm{C}\right)$ affected up to $15.9 \%$ of children. The rate of fever slightly increased when the hexavalent vaccine was co-administered with other paediatric vaccines as compared to the hexavalent vaccine alone $(30.8 \%$ $76.7 \%$ vs $11.0 \%-57.3 \%)$. Toddlers experienced fever with a slightly increased incidence as compared to infants $(31.1 \%$ $52.1 \%$ vs $9.2 \%$ - 46.3\%). uAEs ranged between $17.7 \%$ - 51.1\% for hexavalent vaccine administered alone or concomitantly with other paediatric vaccines. No vaccine-related SAEs were reported for the hexavalent vaccine administered alone, while four vaccine-related SAEs (urticaria, febrile seizure, hypotonic-hyporesponsive episode and Kawasaki disease) were reported when this was co-administered with other paediatric vaccines. The reviewed studies from Europe, published between 2000 2016, showed that hexavalent vaccine was generally well tolerated as primary and booster vaccination.
\end{abstract}

\section{Rezumat}

Orice informație relaționată cu siguranța vaccinurilor influențează încrederea populației în vaccinare. Acest studiu sintetizează datele de siguranță ale vaccinului hexavalent (toxoid diphtheric-tetanic-pertussis, virus poliomielitic inactivat, antigene împotriva hepatitei B și polizaharide de Haemophilus influenzae tip B) publicate în studiile clinice randomizate, efectuate în Europa și publicate în perioada 2000 - 2016. Au fost incluse studii în care vaccinul hexavalent a fost administrat singur sau în asociere cu alte vaccinuri. Au fost evaluate evenimentele adverse solicitate, nesolicitate şi cele grave. Au fost incluse în analiză 15 studii, cu un număr total de 6.618 de copii cu vârsta cuprinsă între 2 și 24 de luni. Reacțiile locale de gradul 3 au variat între 1,1\% - 15,5\% pentru roșeață, $0,4 \%-7,4 \%$ pentru durere și $1,4 \%-9,7 \%$ pentru indurație. Dintre simptomele generale, cele mai frecvent raportate au fost iritabilitatea și febra. Incidența febrei a variat între 11,0\% - 57,3\%, iar febra de gradul $3\left(>39,5^{\circ} \mathrm{C}\right)$ a afectat până la $15,9 \%$ din copii. Frecvența febrei a fost ușor crescută atunci când vaccinul hexavalent a fost administrat împreună cu alte vaccinuri comparativ cu vaccinul hexavalent administrat singur (30,8\% $76,7 \%$ vs $11,0 \%-57,3 \%)$. Febra a fost raportată cu o frecvență mai mare la copiii mici, comparativ cu sugarii $(31,1 \%-52,1 \%$ vs 9,2\% - 46,3\%). Evenimentele adverse nesolicitate au variat între 17,7\% - 51,1\% atunci când vaccinul hexavalent a fost administrat singur sau împreună cu alte vaccinuri. $\mathrm{Nu}$ s-au raportat evenimente adverse grave corelate cu vaccinarea, atunci când vaccinul hexavalent a fost administrat singur. 4 evenimente adverse grave (urticarie, convulsie febrilă, episod hipotonichiporeactiv, boala Kawasaki) corelate cu vaccinarea au fost raportate atunci când vaccinul hexavalent a fost administrat împreună cu alte vaccinuri. Rezultatele celor 15 studii realizate în Europa și publicate în perioada $2000-2016$ au arătat că vaccinul hexavalent administrat în vaccinare primară și rapel a fost bine tolerat.

Keywords: hexavalent vaccine, safety, primary vaccination, booster vaccination

\section{Introduction}

Vaccines save up to 3 million children's lives annually [42]. However, like any medicine, vaccines can cause adverse reactions (ARs). The most commonly occurring ARs are represented by local site reactions such as pain, redness or swelling, and systemic reactions like fever [5].

Any vaccine safety issue may lead to vaccine hesitancy. A coincidental temporal relationship between vaccines and adverse events (AEs) 
FARMACIA, 2018, Vol. 66, 5

following immunization can decrease public confidence in vaccines [21, 28]. For example, concerns emerged about a possible link between measles-mumps-rubella (MMR) vaccine and autism in the late 1990s [39]. The vaccination rate decreased and as a consequence measles outbreaks occurred. During 2011, more than 26,000 cases of measles were reported in Europe [41]; nowadays the number is smaller but still significant (almost 5,000 cases during February 2016 - January 2017) [43]. Yet, a review of 64 studies, involving 14,700,000 children, had found no link between MMR vaccine and autism [7]. In addition, various controversial AEs have been reported for the hepatitis B vaccine as well, such as rheumatoid arthritis, diabetes, chronic fatigue syndrome, lymphoblastic leukaemia and multiple sclerosis. Epidemiological studies did not confirm an increased incidence of these AEs in the population vaccinated with the hepatitis B vaccine [8, 25, 44]. Another important concern regarding vaccines is represented by the multiple injections received by children at a vaccination visit. Wallace et al evidenced that the parental concern about multiple injections at the same vaccination visit being related to more AEs influences their acceptance [40]. Combination vaccines had been therefore developed to reduce the number of injections and to simplify the immunization schedules. Although, combination vaccines have many advantages, misconceptions occurred about their safety profile too [19].

Larson et al observed a low confidence in vaccines in many European countries due to the distrust in the vaccines safety [21].

The goal of this review was to present the safety profile reported in the randomized controlled trials (RCTs) published between 2000 - 2016 assessing the hexavalent vaccines available on the market or under development in Europe. The hexavalent vaccine combines the diphtheria-tetanus-acellular pertussis (DTPa) toxoid with inactivated poliomyelitis virus (IPV), antigens against hepatitis B (HBV) and Haemophilus influenzae type B (Hib) polysaccharide.

\section{Materials and Methods}

\section{Selection criteria}

We searched the PubMed database for English language scientific papers, published between January 2000 and November 2016. We used the following keywords: dtpa hbv ipv hib, dtpa hbv ipv hib safety, hexavalent vaccine, Infanrix safety, Infanrix hexa, Hexavac, Hexyon, Hexacima, Hexaxim. We selected randomized controlled trials (RCTs) that assessed the safety profile of the hexavalent vaccine administered alone or together with other paediatric vaccines. We excluded the ones that published the information as figures only and did not have any additional information on the data included in the figures. In addition, reference lists of the selected publications for the review were screened to identify other potential eligible articles. Additional information available from the study reports published on the clinicaltrials.gov [34], clinicaltrial-registers.eu [9] and on gsk-clinicalstudyregister.com [18] was also included where needed information was missing from the papers. In this review, we reported results from RCTs, conducted in European countries that assessed the safety profile of different formulation of hexavalent vaccine in healthy children up to two years of age. The safety data were reviewed for vaccines that were marketed or under development between 2000 - 2016 .

The vaccines safety in the selected RCTs was monitored with diary cards except the DTPa5-HBVIPV-Hib vaccine. Solicited AEs were represented by local reactions (redness, swelling, pain) and systemic events (fever, decreased appetite, increased sleep, irritability). Unsolicited AEs (uAEs) were recorded for up to one month post-vaccination. In all studies, serious AEs (SAEs) were reported during the entire period of the study. Most studies defined a SAE as an adverse experience that resulted in one of the following conditions: death, immediate life-threatening event, permanent or substantial disability, patient hospitalization. We included only the studies where causal relation-ship between the AEs and vaccination was assessed by the investigator.

\section{Results and Discussion}

We included in our review 15 RCTs that met the inclusion criteria: two double blind studies [4, 29], five single blind studies [20,31, 35-37] and eight open studies $[1,2,6,17,27,30,32,45]$. These studies assessed the safety of different formulations of the hexavalent vaccine after primary and/or booster vaccination: 15 RCTs evaluated authorized vaccines (Infanrix ${ }^{\mathrm{TM}}$ hexa, Hexavac ${ }^{\mathrm{TM}}$, Hexaxim $^{\circledR}$ ) of which one study compared the investigational vaccine (DTPa5-HBV-IPV-Hib) with the Infanrix ${ }^{\text {TM }}$ hexa.

A total of 6,618 children were included in the safety analysis of these RCTs: 5,222 children received Infanrix ${ }^{\mathrm{TM}}$ hexa, $472 \mathrm{Hexavac}^{\mathrm{TM}}, 271$ Hexaxim $^{\circledR}$ and 653 DTPa5-HBV-IPV-Hib. The vaccination schedules varied across studies. The composition of hexavalent vaccines administered in RCTs is presented in Table I. Characteristics of included studies and representative demographic characteristics of the children are summarized in Table II. 
Table I

Description of hexavalent vaccines composition administered in RCTs

\begin{tabular}{|c|c|c|c|c|}
\hline Vaccine component & \begin{tabular}{|c|} 
DTPa-HBV-IPV/Hib \\
(Infanrix hexa $^{\text {TM }}$ ) \\
{$[1,2,4,6,17,20,27$} \\
$29-32,35-37,45]$ \\
\end{tabular} & $\begin{array}{c}\text { DTPa-HBV- } \\
\text { IPV-Hib } \\
\left(\begin{array}{c}\text { Hexavac } \\
\text { TM }\end{array}\right) * \\
{[20,31]}\end{array}$ & \begin{tabular}{|c|} 
DTPa5-HBV-IPV- \\
Hib (Sanofi \\
Pasteur MSD) \\
{$[29]$} \\
\end{tabular} & $\begin{array}{c}\text { DTPa-IPV- } \\
\text { HBV-PRP-T } \\
\text { (Hexaxim }^{\circledR} \text { ) } \\
{[37]} \\
\end{array}$ \\
\hline D & $\geq 30 \mathrm{IU}$ & $\geq 20 \mathrm{IU}$ & Not less than $15 \mathrm{Lf}$ & $\geq 20 \mathrm{IU}$ \\
\hline $\mathbf{T}$ & $\geq 40 \mathrm{IU}$ & $\geq 40 \mathrm{IU}$ & Not less than $5 \mathrm{Lf}$ & $\geq 40 \mathrm{IU}$ \\
\hline PT $(\mu \mathrm{g})$ & 25 & 25 & 20 & 25 \\
\hline FHA $(\mu \mathrm{g})$ & 25 & 25 & 20 & 25 \\
\hline PRN ( $\mu g)$ & 8 & - & 3 & - \\
\hline FIM $\geq \mathbf{3 0}$ & - & - & 5 & - \\
\hline HBs $(\mu \mathrm{g})$ & 10 & 5 & 10 & 10 \\
\hline IPV type 1 (D antigen units) & 40 & 40 & 40 & 40 \\
\hline IPV type 2 (D antigen units) & 8 & 8 & 8 & 8 \\
\hline IPV type 3 (D antigen units) & 32 & 32 & 32 & 32 \\
\hline PRP of Hib conjugated to $T$ & $\begin{array}{c}10 \mu \mathrm{g} \text { PRP } \\
20-40 \mu \mathrm{g} \mathrm{T}\end{array}$ & $\begin{array}{l}12 \mu \mathrm{g} \text { PRP } \\
24 \mu \mathrm{g} \mathrm{T}\end{array}$ & - & $\begin{array}{c}12 \mu \mathrm{g} \text { PRP } \\
22-36 \mu \mathrm{g} \mathrm{T}\end{array}$ \\
\hline $\begin{array}{l}\text { PRP of Hib conjugated to } N \text {. meingitidis sero- } \\
\text { group B outer membrane protein (PRP-OMPC) }\end{array}$ & - & - & $\begin{array}{c}3 \mu \mathrm{g} \text { PRP } \\
50 \mu \mathrm{g} \text { OMPC }\end{array}$ & \\
\hline Aluminum (mg) & 0.82 & 0.3 & 0.32 & 0.6 \\
\hline
\end{tabular}

Table II

Characteristics of the RCTs included in the review

\begin{tabular}{|c|c|c|c|c|c|c|c|c|c|}
\hline \multirow[t]{2}{*}{$\begin{array}{c}\text { Author } \\
\text { (year) }\end{array}$} & \multirow[t]{2}{*}{ Country } & \multirow[t]{2}{*}{$\begin{array}{l}\text { Study } \\
\text { design }\end{array}$} & \multirow[t]{2}{*}{$\begin{array}{c}\text { Vaccine } \\
\text { (trade name) }\end{array}$} & \multirow{2}{*}{$\begin{array}{c}\text { Concomitant } \\
\text { vaccine } \\
\text { (trade } \\
\text { name) }\end{array}$} & \multirow{2}{*}{$\begin{array}{l}\text { Number of } \\
\text { subjects } \\
\text { included in } \\
\text { the safety } \\
\text { analysis }\end{array}$} & \multicolumn{2}{|c|}{$\begin{array}{l}\text { Vaccination } \\
\text { schedule }\end{array}$} & \multirow{2}{*}{\begin{tabular}{|c|} 
Age of \\
enrolled \\
children - \\
mean \\
(range)
\end{tabular}} & \multirow{2}{*}{$\begin{array}{c}\text { Ethnicity of } \\
\text { enrolled } \\
\text { children } \\
\text { (\% } \% \\
\text { Caucasian) }\end{array}$} \\
\hline & & & & & & $\begin{array}{c}\text { Infant } \\
\text { schedule } \\
\text { (months } \\
\text { of age) }\end{array}$ & $\begin{array}{l}\text { Toddler } \\
\text { dose } \\
\text { (months } \\
\text { of age) }\end{array}$ & & \\
\hline \multirow[t]{2}{*}{$\begin{array}{c}\text { Aristegui et } \\
\text { al } \\
{[1](2003)}\end{array}$} & \multirow[t]{2}{*}{ Spain } & \multirow[t]{2}{*}{$\begin{array}{l}\text { Open, } \\
\text { randomized, } \\
\text { multicentre, } \\
\text { Phase III }\end{array}$} & $\begin{array}{c}\text { DTPa-HBV- } \\
\text { IPV/Hib } \\
\text { (Infanrix }^{\text {TM }} \\
\text { hexa) }\end{array}$ & - & 120 & \multirow[t]{2}{*}{$2,4,6$} & \multirow[t]{2}{*}{-} & \multirow[t]{2}{*}{$\begin{array}{c}8.7 \text { weeks } \\
(8-11)\end{array}$} & \multirow[t]{2}{*}{ NA } \\
\hline & & & $\begin{array}{c}\text { DTPa-IPV/Hib } \\
\text { (Infanrix }^{\mathrm{TM}}- \\
\text { IPV/Hib) }+ \\
\text { HBV } \\
\left(\text { Engerix }^{\mathrm{TM}} \mathrm{B}\right)\end{array}$ & - & 115 & & & & \\
\hline \multirow[t]{2}{*}{$\begin{array}{c}\text { Avdicova et } \\
\text { al } \\
{[2](2002)}\end{array}$} & \multirow[t]{2}{*}{ Slovakia } & \multirow[t]{2}{*}{$\begin{array}{l}\text { Open, } \\
\text { randomized, } \\
\text { multicentre }\end{array}$} & $\begin{array}{l}\text { DTPa-HBV- } \\
\text { IPV/Hib } \\
\text { (Infanrix }^{\mathrm{TM}} \\
\text { hexa) }\end{array}$ & - & 156 & \multirow[t]{2}{*}{3,5} & \multirow[t]{2}{*}{11} & \multirow[t]{2}{*}{$\begin{array}{c}13.2 \\
\text { weeks } \\
(8-20)\end{array}$} & \multirow[t]{2}{*}{ NA } \\
\hline & & & $\begin{array}{c}\text { DTPa-IPV/Hib } \\
\text { (Infanrix }^{\mathrm{TM}}- \\
\text { IPV/Hib) }+ \\
\text { HBV } \\
\left.\text { (Engerix }^{\mathrm{TM}} \mathrm{B}\right)\end{array}$ & - & 156 & & & & \\
\hline \multirow[t]{2}{*}{$\begin{array}{c}\text { Gabutti et al } \\
{[17](2004)}\end{array}$} & \multirow[t]{2}{*}{$\begin{array}{c}\text { Germany, } \\
\text { Italy }\end{array}$} & \multirow[t]{2}{*}{$\begin{array}{c}\text { Open, } \\
\text { randomized, } \\
\text { multicentre, } \\
\text { Phase III }\end{array}$} & $\begin{array}{l}\text { DTPa-HBV- } \\
\text { IPV/Hib } \\
\text { (Infanrix }^{\mathrm{TM}} \\
\text { hexa) }\end{array}$ & - & 218 & \multirow[t]{2}{*}{3,5} & \multirow[t]{2}{*}{11} & \multirow[t]{2}{*}{$\begin{array}{c}13.3 \\
\text { weeks } \\
(9-17)\end{array}$} & 96.8 \\
\hline & & & $\begin{array}{l}\text { DTPa-HBV- } \\
\text { IPV (Infanrix } \\
\text { penta) }+ \text { Hib }\end{array}$ & - & 216 & & & & 99.1 \\
\hline \multirow[t]{2}{*}{$\begin{array}{c}\text { Saenger et } \\
\boldsymbol{a l}^{\boldsymbol{a}} \\
{[27](2005)}\end{array}$} & \multirow[t]{2}{*}{ Germany } & \multirow[t]{2}{*}{$\begin{array}{c}\text { Open, } \\
\text { randomized, } \\
\text { multicentre, } \\
\text { Phase III }\end{array}$} & $\begin{array}{l}\text { DTPa-HBV- } \\
\text { IPV/Hib } \\
\text { (Infanrix }^{\mathrm{TM}} \\
\text { hexa) }\end{array}$ & - & 688 & \multirow[t]{2}{*}{-} & \multirow[t]{2}{*}{$12-24$} & \multirow[t]{2}{*}{$\begin{array}{c}15.3 \\
\text { months }\end{array}$} & \multirow[t]{2}{*}{$>95$} \\
\hline & & & $\begin{array}{c}\text { DTPa-IPV/Hib } \\
\text { (Infanrix }^{\text {TM }} \\
\text { IPV/Hib) }+\end{array}$ & - & 227 & & & & \\
\hline
\end{tabular}


FARMACIA, 2018, Vol. 66, 5

\begin{tabular}{|c|c|c|c|c|c|c|c|c|c|}
\hline \multirow[t]{4}{*}{$\begin{array}{c}\text { Author } \\
\text { (year) }\end{array}$} & \multirow[t]{4}{*}{ Country } & \multirow[t]{4}{*}{$\begin{array}{l}\text { Study } \\
\text { design }\end{array}$} & \multirow[t]{2}{*}{$\begin{array}{c}\text { Vaccine } \\
\text { (trade name) }\end{array}$} & \multirow{2}{*}{$\begin{array}{c}\text { Concomitant } \\
\text { vaccine } \\
\text { (trade } \\
\text { name) }\end{array}$} & \multirow{2}{*}{$\begin{array}{c}\text { Number of } \\
\text { subjects } \\
\text { included in } \\
\text { the safety } \\
\text { analysis }\end{array}$} & \multicolumn{2}{|c|}{$\begin{array}{c}\text { Vaccination } \\
\text { schedule }\end{array}$} & \multirow{4}{*}{$\begin{array}{c}\text { Age of } \\
\text { enrolled } \\
\text { children - } \\
\text { mean } \\
\text { (range) }\end{array}$} & \multirow{4}{*}{$\begin{array}{c}\begin{array}{c}\text { Ethnicity of } \\
\text { enrolled } \\
\text { children } \\
(\%\end{array} \\
\text { Caucasian) }\end{array}$} \\
\hline & & & & & & \begin{tabular}{|c|}
$\begin{array}{c}\text { Infant } \\
\text { schedule } \\
\text { (months } \\
\text { of age) }\end{array}$ \\
\end{tabular} & $\begin{array}{c}\begin{array}{c}\text { Toddler } \\
\text { dose } \\
\text { (months } \\
\text { of age) }\end{array} \\
\end{array}$ & & \\
\hline & & & $\begin{array}{c}\mathrm{HBV} \\
\left(\text { Engerix }^{\mathrm{TM}} \mathrm{B}\right)\end{array}$ & & & & & & \\
\hline & & & $\begin{array}{c}\text { DTPa-IPV/Hib } \\
\text { (Infanrix }^{\text {TM }} \\
\text { IPV/Hib) }\end{array}$ & - & 223 & & & & \\
\hline \multirow[t]{2}{*}{$\begin{array}{c}\text { Tichmann } \\
\text { et al } \\
{[31](2005)}\end{array}$} & \multirow[t]{2}{*}{ Germany } & \multirow[t]{2}{*}{$\begin{array}{c}\text { Single blind, } \\
\text { randomized, } \\
\text { multicentre, } \\
\text { Phase IV }\end{array}$} & \begin{tabular}{|c} 
DTPa-HBV- \\
IPV/Hib \\
(Infanrix $^{\mathrm{TM}}$ \\
hexa)
\end{tabular} & - & 235 & \multirow[t]{2}{*}{$2,4,6$} & \multirow[t]{2}{*}{-} & 8.7 weeks & 97.0 \\
\hline & & & $\begin{array}{c}\text { DTPa-HBV- } \\
\text { IPV-Hib } \\
\left(\text { Hexavac }^{\mathrm{TM}}\right)\end{array}$ & - & 224 & & & 8.7 weeks & 93.8 \\
\hline \multirow[t]{2}{*}{$\begin{array}{l}\text { Tichmann } \\
\text { et al } \\
{[30](2006)}\end{array}$} & \multirow[t]{2}{*}{ Germany } & \multirow[t]{2}{*}{$\begin{array}{c}\text { Open, } \\
\text { randomized, } \\
\text { multicentre }\end{array}$} & $\begin{array}{l}\text { DTPa-HBV- } \\
\text { IPV/Hib } \\
\text { (Infanrix }^{\text {TM }} \\
\text { hexa) }^{\mathrm{b}}\end{array}$ & - & 166 & \multirow[t]{2}{*}{-} & \multirow[t]{2}{*}{$11-23$} & \multirow[t]{2}{*}{$\begin{array}{c}14.6 \\
\text { months } \\
(12-19)\end{array}$} & 96.4 \\
\hline & & & $\begin{array}{l}\text { DTPa-HBV- } \\
\text { IPV/Hib } \\
\text { (Infanrix }^{\text {TM }} \\
\text { hexa) }^{\text {c }}\end{array}$ & - & 163 & & & & 95.7 \\
\hline \multirow[t]{2}{*}{$\begin{array}{c}\text { Kilpi et al } \\
{[20](2009)}\end{array}$} & \multirow[t]{2}{*}{$\begin{array}{l}\text { Finland, } \\
\text { Italy, } \\
\text { Sweden }\end{array}$} & \multirow[t]{2}{*}{$\begin{array}{l}\text { Single blind, } \\
\text { randomized, } \\
\text { multicentre, } \\
\text { Phase IV }\end{array}$} & $\begin{array}{l}\text { DTPa-HBV- } \\
\text { IPV/Hib } \\
\text { (Infanrix }^{\text {TM }} \\
\text { hexa) }\end{array}$ & - & 246 & \multirow[t]{2}{*}{3,5} & \multirow[t]{2}{*}{$11-12$} & $\begin{array}{c}12.1 \\
\text { weeks }\end{array}$ & 97.6 \\
\hline & & & $\begin{array}{c}\text { DTPa-HBV- } \\
\text { IPV-Hib } \\
\left(\text { Hexavac }^{\mathrm{TM}}\right)\end{array}$ & - & 248 & & & $\begin{array}{c}12.3 \\
\text { weeks }\end{array}$ & 98.8 \\
\hline \multirow[t]{2}{*}{$\begin{array}{c}\text { Tichmann } \\
\text { et al } \\
{[32](2005)}\end{array}$} & \multirow[t]{2}{*}{ Germany } & \multirow[t]{2}{*}{$\begin{array}{c}\text { Open, } \\
\text { randomized, } \\
\text { multicentre, } \\
\text { Phase IV }\end{array}$} & $\begin{array}{l}\text { DTPa-HBV- } \\
\text { IPV/Hib } \\
\text { (Infanrix }^{\text {TM }} \\
\text { hexa) }\end{array}$ & $\begin{array}{c}\text { PCV7 } \\
\left(\text { Prevenar }^{\mathbb{R}}\right)\end{array}$ & 175 & \multirow[t]{2}{*}{$2,3,4$} & \multirow[t]{2}{*}{$\sim 13$} & $\begin{array}{c}11.5 \\
\text { weeks }\end{array}$ & \multirow[t]{2}{*}{95.0} \\
\hline & & & $\begin{array}{l}\text { DTPa-HBV- } \\
\text { IPV/Hib } \\
\text { (Infanrix }^{\text {TM }} \\
\text { hexa) }\end{array}$ & & 170 & & & & \\
\hline \multirow[t]{2}{*}{$\begin{array}{c}\text { van der } \\
\text { Bergh et al } \\
{[36](2011)}\end{array}$} & \multirow[t]{2}{*}{$\begin{array}{c}\text { The } \\
\text { Netherlands }\end{array}$} & \multirow[t]{2}{*}{$\begin{array}{l}\text { Single blind, } \\
\text { randomized, } \\
\text { single-centre }\end{array}$} & $\begin{array}{c}\text { DTPa-HBV- } \\
\text { IPV/Hib } \\
\text { (Infanrix }^{T M} \\
\text { hexa) }\end{array}$ & \multirow[t]{2}{*}{$\begin{array}{c}\text { PHiD-CV } \\
\text { (Synflorix }^{\mathrm{TM}} \\
)\end{array}$} & 260 & $2,3,4$ & - & 7.4 weeks & 99.2 \\
\hline & & & 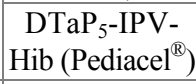 & & 260 & & & 7.6 weeks & 98.1 \\
\hline $\begin{array}{c}\text { van der } \\
\text { Bergh et al } \\
{[35](2016)}\end{array}$ & $\begin{array}{c}\text { The } \\
\text { Netherlands }\end{array}$ & $\begin{array}{l}\text { Single blind, } \\
\text { randomized, } \\
\text { single-centre }\end{array}$ & $\begin{array}{l}\text { DTPa-HBV- } \\
\text { IPV/Hib } \\
\text { (Infanrix }^{\mathrm{TM}} \\
\text { hexa) }\end{array}$ & $\begin{array}{c}\text { PHiD-CV } \\
\text { Synflorix }^{\mathrm{TM}} \\
)\end{array}$ & 257 & - & $11-13$ & $\begin{array}{c}11.0 \\
\text { months }\end{array}$ & 99.2 \\
\hline & & & 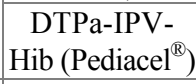 & & 259 & & & $\begin{array}{c}11.1 \\
\text { months }\end{array}$ & 98.1 \\
\hline $\begin{array}{c}\text { Berner et al } \\
{[4]} \\
(2012)\end{array}$ & Germany & $\begin{array}{c}\text { Double } \\
\text { blind, } \\
\text { randomized, } \\
\text { multicentre, }\end{array}$ & $\begin{array}{c}\text { DTPa-IPV- } \\
{\text { Hib } \text { (Pediacel }^{\mathbb{B}} \text { ) }}^{+ \text {HBV }} \\
\left.\text { (Engerix }^{\mathrm{TM}} \mathrm{B}\right)\end{array}$ & $\begin{array}{c}\text { PCV7 } \\
\left(\text { Prevenar }^{(\mathbb{}}\right)\end{array}$ & 422 & - & $11-18$ & $\begin{array}{c}13.6 \\
\text { months } \\
(11.0- \\
19.3)\end{array}$ & NA \\
\hline & & Phase III & $\begin{array}{l}\text { DTPa-HBV- } \\
\text { IPV/Hib } \\
\text { (Infanrix }^{\text {TM }} \\
\text { hexa) }\end{array}$ & & 421 & & & $\begin{array}{c}13.7 \\
\text { months } \\
(11.0- \\
18.8)\end{array}$ & \\
\hline $\begin{array}{c}\text { Vesikari et } \\
\text { al }\end{array}$ & $\begin{array}{l}\text { Sweden, } \\
\text { Finland }\end{array}$ & $\begin{array}{l}\text { Single blind, } \\
\text { randomized, }\end{array}$ & $\begin{array}{l}\text { DTPa-IPV- } \\
\text { HBV-PRP-T }\end{array}$ & \begin{tabular}{|c|} 
PCV13 \\
(Prevenar $^{\mathbb{R}}$ 13)
\end{tabular} & 271 & 3,5 & $11-12$ & 89.6 days & $>94.0$ \\
\hline
\end{tabular}


FARMACIA, 2018, Vol. 66, 5

\begin{tabular}{|c|c|c|c|c|c|c|c|c|c|}
\hline \multirow[t]{2}{*}{$\begin{array}{c}\begin{array}{c}\text { Author } \\
\text { (year) }\end{array}\end{array}$} & \multirow[t]{2}{*}{ Country } & \multirow[t]{2}{*}{$\begin{array}{l}\text { Study } \\
\text { design }\end{array}$} & \multirow[t]{2}{*}{$\begin{array}{c}\text { Vaccine } \\
\text { (trade name) }\end{array}$} & \multirow{2}{*}{$\begin{array}{c}\text { Concomitant } \\
\text { vaccine } \\
\text { (trade } \\
\text { name) }\end{array}$} & \multirow{2}{*}{$\begin{array}{c}\text { Number of } \\
\text { subjects } \\
\text { included in } \\
\text { the safety } \\
\text { analysis }\end{array}$} & \multicolumn{2}{|c|}{$\begin{array}{c}\text { Vaccination } \\
\text { schedule }\end{array}$} & \multirow{2}{*}{$\begin{array}{c}\text { Age of } \\
\text { enrolled } \\
\text { children - } \\
\text { mean } \\
\text { (range) }\end{array}$} & \multirow{2}{*}{$\begin{array}{c}\text { Ethnicity of } \\
\text { enrolled } \\
\text { children } \\
(\% \\
\text { Caucasian) }\end{array}$} \\
\hline & & & & & & \begin{tabular}{|c|}
$\begin{array}{c}\text { Infant } \\
\text { schedule } \\
\text { (months } \\
\text { of age) }\end{array}$ \\
\end{tabular} & $\begin{array}{c}\text { Toddler } \\
\text { dose } \\
\text { (months } \\
\text { of age) }\end{array}$ & & \\
\hline \multirow[t]{2}{*}{ [37] (2017) } & & \multirow{2}{*}{$\begin{array}{c}\text { multicentre, } \\
\text { Phase III }\end{array}$} & $\left(\right.$ Hexaxim $\left.^{(B)}\right)$ & & & & & & \\
\hline & & & $\begin{array}{l}\text { DTPa-HBV- } \\
\text { IPV/Hib } \\
\text { (Infanrix } \\
\text { hexa) }\end{array}$ & & 275 & & & 89 days & \\
\hline \multirow{2}{*}{$\begin{array}{c}\text { Silfverdal et } \\
\text { al } \\
{[29](2016)}\end{array}$} & \multirow{2}{*}{$\begin{array}{l}\text { Sweden, } \\
\text { Italy, } \\
\text { Finland }\end{array}$} & \multirow{2}{*}{$\begin{array}{c}\text { Double } \\
\text { blind, } \\
\text { randomized, } \\
\text { multicentre, } \\
\text { Phase III }\end{array}$} & $\begin{array}{l}\text { DTPa5-HB- } \\
\text { IPV-Hib }\end{array}$ & \multirow{2}{*}{$\begin{array}{c}\text { PCV13 } \\
\left(\text { Prevenar }^{\mathbb{B}} \text { 13) }\right. \\
\text { RV1 } \\
\left(\text { Rotarix }^{\mathbb{B}}\right) / \\
\text { RV5 } \\
\left(\text { RotaTeq }^{\circledR}\right)\end{array}$} & 653 & \multirow[t]{2}{*}{2,4} & \multirow[t]{2}{*}{$11-12$} & $\begin{array}{l}68.0 \text { days } \\
(46-89)\end{array}$ & \multirow[t]{2}{*}{ NA } \\
\hline & & & $\begin{array}{c}\text { DTPa-HBV- } \\
\text { IPV/Hib } \\
\text { (Infanrix }^{\text {TM }} \\
\text { hexa) }\end{array}$ & & 659 & & & $\begin{array}{l}68.1 \text { days } \\
(46-89)\end{array}$ & \\
\hline \multirow[t]{3}{*}{$\begin{array}{c}\text { Zepp et al } \\
{[45](2007)}\end{array}$} & \multirow[t]{3}{*}{ Germany } & \multirow[t]{3}{*}{$\begin{array}{c}\text { Open, } \\
\text { randomized, } \\
\text { multicentre }\end{array}$} & $\begin{array}{l}\text { DTPa-HBV- } \\
\text { IPV/Hib } \\
\text { (Infanrix } \\
\text { hexa) }\end{array}$ & $\begin{array}{l}\text { MMRV } \\
\text { (Priorix- } \\
\text { Tetra }^{\circledR} \text { ) }\end{array}$ & 150 & \multirow[t]{3}{*}{-} & \multirow[t]{3}{*}{$12-23$} & $\begin{array}{c}13.7 \\
\text { months }\end{array}$ & \multirow[t]{3}{*}{95.3} \\
\hline & & & - & $\begin{array}{l}\text { MMRV } \\
\text { (Priorix- } \\
\text { Tetra }^{\circledR} \text { ) }\end{array}$ & 150 & & & & \\
\hline & & & $\begin{array}{l}\text { DTPa-HBV- } \\
\text { IPV/Hib } \\
\text { (Infanrix } \\
\text { hexa) }\end{array}$ & - & 150 & & & & \\
\hline \multirow[t]{3}{*}{$\begin{array}{c}\text { Deichmann } \\
\text { et al } \\
{[6](2015)}\end{array}$} & \multirow[t]{3}{*}{$\begin{array}{l}\text { Germany, } \\
\text { Italy }\end{array}$} & \multirow[t]{3}{*}{$\begin{array}{c}\text { Open, } \\
\text { randomized, } \\
\text { multicentre }\end{array}$} & $\begin{array}{l}\text { DTPa-HBV- } \\
\text { IPV/Hib } \\
\text { (Infanrix }^{\text {TM }} \\
\text { hexa) }\end{array}$ & $\begin{array}{c}\text { MMRV } \\
\left(\text { ProQuad }^{\circledR}\right)\end{array}$ & 474 & \multirow[t]{3}{*}{-} & \multirow[t]{3}{*}{$12-24$} & $\begin{array}{c}13.6 \\
\text { months }\end{array}$ & \multirow[t]{3}{*}{ NA } \\
\hline & & & - & $\begin{array}{c}\text { MMRV } \\
\left(\text { ProQuad }^{\circledR}\right)\end{array}$ & 234 & & & $\begin{array}{c}13.4 \\
\text { months }\end{array}$ & \\
\hline & & & $\begin{array}{l}\text { DTPa-HBV- } \\
\text { IPV/Hib } \\
\text { (Infanrix } \\
\text { hexa) }\end{array}$ & - & 239 & & & $\begin{array}{c}13.5 \\
\text { months }\end{array}$ & \\
\hline
\end{tabular}

a, we included in review study A, a pre-marketing trial; study B was a post-marketing trial and assessed the less frequent adverse events and was not included in our review; ${ }^{\mathrm{b}}$, children primary vaccinated with Infanrix ${ }^{\mathrm{TM}}$ hexa; ${ }^{\mathrm{c}}$, , children primary vaccinated with Hexavac ${ }^{\mathrm{TM}}$; NA, data not available; DTPa, diphtheria toxoid, tetanus toxoid, acellular pertussis toxoid; HBV, antigens against hepatitis B; IPV, inactivated poliomyelitis virus; Hib, Haemophilus influenzae type b polysaccharide; PCV, PHiD-CV, pneumococcal conjugate vaccine; RV, rotavirus vaccine; MMRV, measles-mumps-rubella-varicella vaccine.

\section{Solicited adverse events}

Solicited AEs were collected either for $4[1,2,17$, $20,27,30-32,35,36], 5$ [29] or 7 [37] days after each vaccination. Fever was reported within 4 days and 6 weeks after vaccination in the Berner et al study and Zepp et al study, respectively [4, 45]. In these studies, the other solicited AEs were reported within 7 and 4 days, respectively. The solicited local reactions and the solicited systemic reactions were reported within 5 days and 29 days after vaccination, respectively in the Deichmann et al study [6].

The incidence of local reactions for the hexavalent vaccine ranged between $26.4 \%-61.9 \%$ for redness, $17.0 \%$ - $61.1 \%$ for pain and $17.5 \%$ - $64.6 \%$ for swelling. Grade 3 local reactions varied between $1.1 \%-15.5 \%$ for redness, $0.4 \%-7.4 \%$ for pain and $1.4 \%-9.7 \%$ for swelling. In papers reporting large swelling $[4,27,30,35,37]$ (defined as swelling $>5$ $\mathrm{mm}$ in diameter and/or any noticeable diffuse swelling or increase of the circumference of injected limb) we found the incidence of AE to be between $1.55 \%-7.4 \%$, while extensive limb swelling (defined as swelling extending from the injection site beyond one or both adjacent joints) was reported for one subject.

The co-administration of the hexavalent vaccine with other paediatric vaccines resulted in a slightly increased incidence of fever compared with the hexavalent vaccine alone $(30.8 \%-76.7 \%$ vs $11.0 \%$ - 57.3\%). Grade 3 fever ranged between $0.4 \%$ $2.6 \%$ in the co-administered group and between $0.0 \%-15.9 \%$ in the hexavalent group. This data is reflected in Table III and Table IV. Toddlers experienced fever with a slightly increased incidence as compared to infants when hexavalent vaccine was 
administered with/without other paediatric vaccines (31.1\% - 52.1\% vs 9.2\% - 46.3\%). The incidence of solicited general symptoms at each dose is presented in Table V.

Among the most frequently reported solicited general symptom was irritability when the hexavalent vaccine was administered alone or together with other paediatric vaccines $(1.3 \%-59.6 \%$ and $1.3 \%-65.3 \%)$. The incidence of solicited AEs of hexavalent vaccine administered alone or coadministered with other paediatric vaccines is presented in Table III and Table IV, respectively. The reactogenicity was presented per subject in Vesikari et al and Silfverdal et al studies. In those studies, the general symptoms ranged between $89.4 \%$ - 96.7\% for irritability, $69.0 \%$ - $99.7 \%$ for fever, $79.2 \%$ $86.1 \%$ for drowsiness and $62.2 \%-67.2 \%$ for decreased appetite.

Unsolicited adverse events

uAEs were represented by local reactions that extended after the follow-up period, respiratory tract disorders (upper respiratory infection, rhinitis, otitis media, bronchitis, nasopharyngitis, cough), gastrointestinal disorders (diarrhoea, gastroenteritis, vomiting), pyrexia, conjunctivitis, eczema and erythematous rash, injection site rash, hematoma, granuloma $[1,2,4,20,27,31,32,36]$. The percentage of subjects with uAEs ranged between $33.7 \%$ - 51.1\% while vaccination related uAEs were $0.6 \%-16.3 \%$ when the hexavalent vaccine was administered alone [17, 20, 27, 30-32].

The incidence of uAEs ranged between $17.7 \%$ $48.5 \%$ after the co-administration of the hexavalent vaccine and the pneumococcal conjugate vaccine (PCV) [4, 32, 35-37]. One febrile convulsion and one convulsion were reported after the co-administration of the hexa-valent vaccine, PCV and the rotavirus vaccine (RV) [29].

Overall, no participant and respectively $2 \%$ of participants reported at least one vaccine related uAE after administration of hexavalent vaccine together with the measles-mumps-rubella-varicella vaccine (MMRV) and the hexavalent vaccine alone. No significant difference was observed regarding the incidence of generalized rash in the coadministered group $v s$. hexavalent alone group (12.0\% vs 9.3\%) [45]. However, Deichmann et al found a higher rate of non-injection site rash in the co-administered group than in the hexavalent group $(13.5 \%$ vs $3.8 \%)[6]$.

Table III

Reactogenicity of hexavalent vaccine. Incidence of solicited local and general symptoms during 4 - 7 day follow-

\begin{tabular}{|c|c|c|c|c|c|c|c|c|}
\hline \multirow[t]{2}{*}{ Study } & \multirow[t]{2}{*}{ Vaccine } & \multicolumn{3}{|c|}{ Local symptoms (overall/Grade $3^{\mathrm{a}}$ ) } & \multicolumn{4}{|c|}{ General symptoms (overall/Grade $3^{\text {a }}$ ) } \\
\hline & & Pain & Redness & Swelling & Fever & $\begin{array}{c}\text { Decreased } \\
\text { appetite }\end{array}$ & $\begin{array}{l}\text { Irritability/ } \\
\text { Fussiness }\end{array}$ & $\begin{array}{c}\text { Increased sleep/ } \\
\text { drowsiness }\end{array}$ \\
\hline \multirow{2}{*}{$\begin{array}{c}\text { Aristegui et al }^{b} \\
{[1]}\end{array}$} & Infanrix ${ }^{\mathrm{TM}}$ hexa & $22.6 / 2.5$ & $30.4 / 1.1$ & $22.6 / 1.4$ & $21.1 / 0.0$ & $25.3 / 0.6$ & $39.7 / 2.8$ & $27.5 / 0.3$ \\
\hline & $\begin{array}{c}\text { Infanrix }{ }^{\mathrm{TM}} \text {-IPV/ } \\
\text { Hib + Engerix }{ }^{\mathrm{TM}} \mathrm{B}\end{array}$ & $\begin{array}{c}16.8 / 1.2 \\
9.9 / 0.3 \\
\end{array}$ & $\begin{array}{l}20.9 / 0.3 \\
11.6 / 0.0\end{array}$ & $\begin{array}{r}15.1 / 0.9 \\
5.2 / 0.0 \\
\end{array}$ & $12.2 / 0.3$ & $21.4 / 0.3$ & $32.8 / 2.0$ & $29.0 / 0.3$ \\
\hline \multirow{2}{*}{$\begin{array}{c}\text { Avdicova et al } \\
\text { [2] }\end{array}$} & Infanrix $^{\mathrm{TM}}$ hexa & $19.2 / 0.4$ & $31.9 / 3.0$ & $17.5 / 3.2$ & $11.0 / 0.2$ & NA & $17.5 / 0.0$ & NA \\
\hline & $\begin{array}{c}\text { Infanrix }{ }^{\mathrm{TM}}-\mathrm{IPV} / \\
\mathrm{Hib}+\text { Engerix }^{\mathrm{TM}} \mathrm{B}\end{array}$ & $\begin{array}{l}17.2 / 0.2 \\
10.1 / 0.0\end{array}$ & $\begin{array}{l}30.6 / 1.3 \\
18.1 / 0.2\end{array}$ & $\begin{array}{r}15.3 / 1.7 \\
7.3 / 0.9\end{array}$ & $9.1 / 0.6$ & NA & $14.0 / 0.0$ & NA \\
\hline \multirow{2}{*}{$\begin{array}{c}\text { Gabutti et al } \\
{[17]}\end{array}$} & Infanrix $^{\mathrm{TM}}$ hexa & $18.7 / 1.6$ & $26.4 / 3.8$ & $22.3 / 4.1$ & $29.3 / 0.9$ & $18.1 / 0.6$ & $29.8 / 0.9$ & $25.8 / 0.6$ \\
\hline & $\begin{array}{c}\text { Infanrix }{ }^{\mathrm{TM}} \text { penta }+ \\
\text { Hib }\end{array}$ & $\begin{array}{l}14.8 / 1.1 \\
13.1 / 0.5\end{array}$ & $\begin{array}{l}22.5 / 1.8 \\
18.0 / 1.3\end{array}$ & $\begin{array}{l}16.9 / 2.9 \\
11.6 / 1.0\end{array}$ & $23.4 / 1.3$ & $18.3 / 1.4$ & $31.5 / 4.0$ & $25.0 / 1.6$ \\
\hline \multirow{3}{*}{$\begin{array}{c}\text { Saenger et } a^{b} \\
{[27]}\end{array}$} & Infanrix ${ }^{\mathrm{TM}}$ hexa & $31.2 / 6.4$ & $48.2 / 8.8$ & $30.7 / 8.6$ & $42.2 / 3.6$ & $28.5 / 1.6$ & $34.7 / 2.2$ & $33.7 / 2.0$ \\
\hline & $\begin{array}{c}\text { Infanrix }{ }^{\mathrm{TM}}-\mathrm{IPV} / \\
\mathrm{Hib}+\text { Engerix }^{\mathrm{TM}} \mathrm{B}\end{array}$ & $33.0 / 3.1$ & $42.7 / 6.2$ & $27.3 / 7.5$ & $34.8 / 1.8$ & $24.2 / 0.9$ & $30.8 / 0.9$ & $30.4 / 1.3$ \\
\hline & Infanrix $^{\mathrm{TM}}$-IPV/ Hib & $/ 4.5$ & $43.4 / 10.9$ & $27.1 / 11.8$ & $40.7 / 5.4$ & $26.7 / 1.4$ & $39.8 / 5.0$ & $33.0 / 1.4$ \\
\hline \multirow{2}{*}{$\begin{array}{c}\text { Tichmann et } \\
\boldsymbol{a l}^{c, d}[31]\end{array}$} & Infanrix ${ }^{\mathrm{TM}}$ hexa & $19.5 / 0.9$ & $40.5 / 3.9$ & $27.7 / 6.1$ & $17.7 / 0.0$ & $20.1 / 0.6$ & $35.2 / 1.9$ & $38.3 / 0.9$ \\
\hline & Hexavac $^{\mathrm{TM}}$ & $17.0 / 1.1$ & $31.8 / 2.2$ & $20.4 / 3.6$ & $12.1 / 0.2$ & $21.3 / 0.5$ & $37.2 / 1.9$ & $37.9 / 1.2$ \\
\hline \multirow{2}{*}{$\begin{array}{c}\text { Tichmann et al } \\
{[30]}\end{array}$} & Infanrix ${ }^{\mathrm{TM}}$ hexa $^{\mathrm{e}}$ & $39.2 / 3.6$ & $57.8 / 9.0$ & $40.4 / 7.8$ & $31.3 / 2.4$ & $30.1 / 0.6$ & $38.6 / 1.8$ & $39.2 / 1.2$ \\
\hline & Infanrix ${ }^{\mathrm{TM}}$ hexa $^{\mathrm{f}}$ & $27.0 / 0.6$ & $39.3 / 4.9$ & $28.8 / 3.7$ & $35.0 / 3.1$ & $23.3 / 0.6$ & $40.5 / 0.6$ & $35.6 / 0.6$ \\
\hline \multirow[t]{2}{*}{ Kilpi et al ${ }^{c}$ [20] } & Infanrix ${ }^{\mathrm{TM}}$ hexa & $28.8 / 2.5$ & $39.9 / 8.4$ & $27.1 / 8.2$ & $27.8 / 1.0$ & $22.1 / 1.4$ & $52.9 / 3.0$ & $39.6 / 0.5$ \\
\hline & Hexavac $^{\mathrm{TM}}$ & $44.3 / 7.3$ & $54.0 / 15.5$ & $34.1 / 9.7$ & $35.1 / 0.7$ & $29.2 / 0.6$ & $59.6 / 5.8$ & $46.0 / 1.1$ \\
\hline
\end{tabular}

${ }^{\mathrm{a}}$ Grade 3 symptoms were defined: pain as crying when the limb was moved, redness and swelling $>20 \mathrm{~mm}$ in diameter, fever as rectal temperature $>39.5^{\circ} \mathrm{C}$ except the study [1] where grade 3 fever was defined as rectal temperature $>39.0^{\circ} \mathrm{C}$, drowsiness that prevents normal activity, fussiness as inconsolable or persistent crying, complete loss of appetite; ${ }^{\mathrm{b}}$, results presented to according to protocol cohort; ${ }^{\mathrm{c}}$, results presented to total vaccinated cohort; ${ }^{\mathrm{d}}$, in this study the researcher defined grade 3 fever as rectal temperature $>38.5^{\circ} \mathrm{C}$, but for consistency we presented the $\%$ of doses with the temperature $>39.5^{\circ} \mathrm{C} ;{ }^{\mathrm{e}}$, children primary vaccinated with Infanrix ${ }^{\mathrm{TM}}$ hexa; ${ }^{\mathrm{f}}$, children primary vaccinated with Hexavac ${ }^{\mathrm{TM}}$; NE, not evaluated; NA, data not available. 
Table IV

Reactogenicity of hexavalent vaccine co-administrated with other paediatric vaccines. Incidence of solicited local and general symptoms during 4 - 7 day follow-up period after vaccination ( $\%$ of doses)

\begin{tabular}{|c|c|c|c|c|c|c|c|c|}
\hline \multirow[t]{2}{*}{ Study } & \multirow[t]{2}{*}{ Vaccine } & \multicolumn{3}{|c|}{$\begin{array}{l}\text { Local symptoms } \\
\text { (overall/Grade } 3^{\text {a }} \text { ) }\end{array}$} & \multicolumn{4}{|c|}{$\begin{array}{l}\text { General symptoms } \\
\text { (overall/Grade } 3^{\mathbf{a}} \text { ) }\end{array}$} \\
\hline & & Pain & Redness & Swelling & Fever & $\begin{array}{l}\text { Decreased } \\
\text { appetite }\end{array}$ & $\begin{array}{l}\text { Irritability/ } \\
\text { Fussiness }\end{array}$ & $\begin{array}{c}\text { Increased sleep/ } \\
\text { drowsiness }\end{array}$ \\
\hline \multirow{4}{*}{$\begin{array}{c}\text { Van der Bergh et } \\
\qquad \boldsymbol{a l}^{b}[36]\end{array}$} & \multirow{2}{*}{$\begin{array}{c}\text { Infanrix }{ }^{\mathrm{TM}} \text { hexa }+ \\
\text { Synflorix }^{\mathrm{TM}}\end{array}$} & $44.9 / 5.8$ & $37.8 / 1.2$ & $44.1 / 2.4$ & \multirow[t]{2}{*}{$30.8 / 0.4^{\mathrm{c}}$} & \multirow[t]{2}{*}{$29.9 / 0.5$} & \multirow[t]{2}{*}{$65.3 / 6.5$} & \multirow[t]{2}{*}{$59.9 / 2.1$} \\
\hline & & $47.9 / 5.4$ & $41.3 / 0.9$ & $45.6 / 1.8$ & & & & \\
\hline & \multirow{2}{*}{$\begin{array}{l}\text { Pediacel }^{(B)}+ \\
\text { Synflorix }^{\mathrm{TM}}\end{array}$} & $40.6 / 5.0$ & $37.2 / 3.2$ & $38.9 / 3.2$ & \multirow[t]{2}{*}{$22.2 / 0.3^{\mathrm{c}}$} & \multirow[t]{2}{*}{$29.0 / 0.5$} & \multirow[t]{2}{*}{$63.9 / 5.8$} & \multirow[t]{2}{*}{$54.7 / 1.9$} \\
\hline & & $43.0 / 4.7$ & $43.8 / 2.1$ & $45.6 / 2.3$ & & & & \\
\hline \multirow{4}{*}{$\begin{array}{c}\text { Van der Bergh et } \\
\qquad \boldsymbol{a l}^{b}[35]\end{array}$} & \multirow{2}{*}{$\begin{array}{c}\text { Infanrix }{ }^{\mathrm{TM}} \text { hexa }+ \\
\text { Synflorix }^{\mathrm{TM}}\end{array}$} & $61.1 / 7.4$ & $61.9 / 6.6$ & $64.6 / 8.9$ & \multirow[t]{2}{*}{$39.1 / 2.3$} & \multirow[t]{2}{*}{$36.3 / 2.0$} & \multirow[t]{2}{*}{$65.2 / 4.3$} & \multirow[t]{2}{*}{$51.2 / 2.3$} \\
\hline & & $60.7 / 7.8$ & $61.1 / 4.3$ & $58.8 / 3.9$ & & & & \\
\hline & \multirow{2}{*}{$\begin{array}{l}\text { Pediacel }^{(\mathbb{R}}+ \\
\text { Synflorix }^{\mathrm{TM}}\end{array}$} & $53.9 / 6.2$ & $48.8 / 4.3$ & $45.0 / 3.5$ & \multirow[t]{2}{*}{$38.8 / 2.7$} & \multirow[t]{2}{*}{$32.2 / 0.0$} & \multirow[t]{2}{*}{$62.4 / 3.1$} & \multirow[t]{2}{*}{$45.7 / 1.9$} \\
\hline & & $58.1 / 7.0$ & $52.3 / 5.8$ & $49.2 / 3.1$ & & & & \\
\hline \multirow[t]{3}{*}{ Berner et al ${ }^{d}$ [4] } & Pediacel $^{(\mathbb{B}}+$ Prevenar $^{\mathbb{R}}$ & $63.0 / 3.3$ & $62.8 / 13.7$ & $35.0 / 8.8$ & $63.7 / 7.8$ & $41.5 / 3.3$ & $51.4 / 3.8$ & $39.8 / 1.2$ \\
\hline & + Engerix $^{\mathrm{TM}} \mathrm{B}$ & $33.7 / 0.0$ & $24.4 / 0.0$ & $9.8 / 0.2$ & $28.9 / 5.0^{\mathrm{e}}$ & $24.2 / 2.6^{\mathrm{e}}$ & $29.7 / 1.2^{\mathrm{e}}$ & $25.1 / 0.5^{\mathrm{e}}$ \\
\hline & $\begin{array}{c}\text { Infanrix }^{\mathrm{TM}} \text { hexa }+ \\
\text { Prevenar }^{\circledR}\end{array}$ & $63.7 / 3.3$ & $68.4 / 13.3$ & $35.9 / 7.4$ & $\begin{array}{l}65.0 / 6.0 \\
19.4 / 3.7^{\mathrm{e}}\end{array}$ & $\begin{array}{l}45.5 / 3.8 \\
18.3 / 2.4^{\mathrm{e}}\end{array}$ & $\begin{array}{l}55.2 / 1.7 \\
18.8 / 1.2^{\mathrm{e}}\end{array}$ & $\begin{array}{l}40.0 / 2.1 \\
10.6 / 0.7^{\mathrm{e}}\end{array}$ \\
\hline \multirow[t]{3}{*}{ Zepp et al ${ }^{d}$} & Infanrix $^{\mathrm{TM}}$ hexa + & $\begin{array}{l}26.0 / 2.0 \\
17.3 / 13\end{array}$ & $\begin{array}{l}44.0 / 8.7 \\
31.3 / 07\end{array}$ & $\begin{array}{l}31.3 / 4.7 \\
173 / 0.7\end{array}$ & $76.7 / 18.0$ & NA & NA & NA \\
\hline & $\begin{array}{l}\text { Priorix-Tetra } \\
\text { Priorix-Tetra }\end{array}$ & \begin{tabular}{|l}
$17.3 / 1.3$ \\
$14.0 / 0.0$
\end{tabular} & $\frac{31.3 / 0.1}{27.3 / 0.0}$ & $\frac{1 / .3 / 0.1}{9.3 / 0.0}$ & $74.7 / 19.3$ & NA & NA & NA \\
\hline & Infanrix ${ }^{\mathrm{TM}}$ hexa & $29.3 / 4.0$ & $50.7 / 9.3$ & $36.7 / 9.3$ & $48.0 / 3.3$ & NA & NA & NA \\
\hline \multirow[t]{3}{*}{$\begin{array}{c}\text { Deichmann et al } \\
{[6]}\end{array}$} & $\begin{array}{c}\text { Infanrix }{ }^{\mathrm{TM}} \text { hexa }+ \\
\text { ProQuad }^{\mathbb{B}}\end{array}$ & $\begin{array}{l}39.0 \\
20.9\end{array}$ & $\begin{array}{l}49.8 \\
16.7\end{array}$ & $\begin{array}{c}38.0 \\
9.7\end{array}$ & $69.3 / 22.6^{\mathrm{f}}$ & $\mathrm{NE}$ & $1.3^{\mathrm{f}}$ & $\mathrm{NE}$ \\
\hline & ProQuad $^{(B)}$ & 14.1 & 10.7 & 2.6 & $61.1 / 20.5^{\mathrm{f}}$ & $\mathrm{NE}$ & - & $\mathrm{NE}$ \\
\hline & Infanrix $^{\mathrm{TM}}$ hexa & 35.1 & 52.7 & 38.9 & $57.3 / 15.9^{f}$ & $\mathrm{NE}$ & $1.3^{\mathrm{f}}$ & $\mathrm{NE}$ \\
\hline
\end{tabular}

, Grade 3 symptoms were defined: pain as crying when the limb was moved, redness and swelling with a diameter $>20 \mathrm{~mm}$ in the study [45], $>30 \mathrm{~mm}$ in studies $[35,36]$ and $\geq 5 \mathrm{~cm}$ in the study [4], fever as temperature $>39.5^{\circ} \mathrm{C}$, drowsiness that prevents normal activity, fussiness as inconsolable or persistent crying, complete loss of appetite; ${ }^{b}$, results presented to total vaccinated cohort; ${ }^{c}$, in these studies the researcher defined Grade 3 fever as rectal temperature $>40.0^{\circ} \mathrm{C}$, but for consistency we presented the $\%$ of doses with the temperature $>39.5^{\circ} \mathrm{C} ;{ }^{\mathrm{d}}$, results presented to according to protocol cohort; ${ }^{\mathrm{e}}$, because Engerix ${ }^{\mathrm{TM}} \mathrm{B}$ vaccine was administered 1 month after Pediacel ${ }^{\circledR}$ and Prevenar ${ }^{\circledR}$, the general symptoms were assessed twice for both groups; ${ }^{\mathrm{f}}$, systemic events were collected 29 days post vaccination; NE, not evaluated; NA, data not available; Prevenar ${ }^{\mathbb{R}}$, Prevenar ${ }^{\circledR} 13$, pneumococcal conjugate vaccine; Rotarix ${ }^{\circledR}$, Rotateq ${ }^{\mathbb{R}}$, rotavirus vaccine; Priorix $^{\circledR}$-Tetra, ProQuad ${ }^{\circledR}$, measles-mumps-rubella-varicella vaccine.

\section{Serious adverse events}

Across the studies 81 SAEs were reported for a total of 2,859 children which received the hexavalent vaccine alone. None were considered by the investigator to be causally related to vaccination $[2,6$, 17, 20, 27, 30-32]. Aristegui et al reported 4 SAEs for all children included in the safety cohort for the hexavalent and pentavalent vaccine while none of these were causally related to vaccination [1].

Overall, 108 SAEs were reported after the coadministration of the hexavalent vaccine and another paediatric vaccine [4, 6, 29, 32, 35-37, 45]. Four SAEs were considered by the investigators to be related to vaccination (febrile seizure, hypotonichypo-responsive episode, urticaria and Kawasaki disease) [4, 29, 36, 37].

No fatal SAEs were reported in the studies included in our review.

The hexavalent vaccine is used in Europe for more than 16 years and data over this period showed a favourable benefit/risk balance. Three hexavalent vaccines are authorized nowadays in the European Union: Infanrix ${ }^{\mathrm{TM}}$ hexa, Hexyon ${ }^{\circledR} /$ Hexacima $^{\circledR}$ and Vaxelis $^{\circledR}$. Hexavac ${ }^{\mathrm{TM}}$ was authorized for 5 years, but withdrawn due the low immunogenicity against hepatitis B $[10,11,13,14,16]$.

Our review showed that the solicited local reactions reported after the use of hexavalent vaccine were mostly mild in intensity. Redness was the most frequently reported grade 3 solicited local reaction, followed by swelling. Extensive limb swelling, which was observed after booster doses of DTPa vaccines, was reported by one subject across RCTs included in our review. However, a narrative review on DTP containing vaccines have found an incidence of extensive limb swelling of $2 \%-6 \%$ [26].

Across the trials included in our review, fever seemed to be more frequently reported in toddlers than in infants $(31.1 \%$ - $52.1 \%$ vs $9.2 \%-46.3 \%)$. This finding is consistent with the results of a large study $(28.5 \%$ vs $9.2 \%)$ and is included in the Product Information (PI) of Infanrix ${ }^{\mathrm{TM}}$ hexa $[11,22]$. Grade 3 fever was reported after up to $16 \%$ of the doses for the hexavalent vaccine in the RCTs included in our review. In the Macias et al large-scale $(\mathrm{n}=1,422)$ RCT conducted in Latin America, that assessed the safety of the hexavalent vaccine administered at 2 , 4 and 6 months of age, only $4 \%$ of subjects reported severe fever [23]. 
FARMACIA, 2018, Vol. 66, 5

The majority of reported uAEs were not related to vaccine administration. The most commonly vaccine related uAEs were local reactions which extended after the 4 - 7 days follow-up period. Macias et al found a similar incidence of uAEs: $5.7 \%$ of children experienced injection-site nodules out of $10.8 \%$ subjects with uAEs [23].

None of the reported SAEs were considered related to the hexavalent vaccine when this was administrated alone. Nevertheless, an older review of 7 RCTs on Hexavac ${ }^{\mathrm{TM}}$, involving 3,905 infants and 4,437 toddlers, found 8 vaccine related SAEs reported along the studies: 5 SAEs after primary vaccination (high-pitched crying, hypotonic-hyporesponsive episode, intussusception, pyelonephritis, influenza-like syndrome associated with gastroenteritis) and 3 SAEs after booster doses (febrile convulsions) [24]. Furthermore, a hypotonic-hyporesponsive episode was reported for Hexaxim ${ }^{\circledR}$ too, in the Macias et al study [23].

Fatal SAEs or sudden unexpected deaths (SUD) were not reported in the reviewed studies. However, SUD is a rare SAE highly improbable to be detected in RCTs due the small number of children that are included in these studies. An analysis based on national birth cohort data in Germany assessed the incidence of SUD in relation with the hexavalent vaccine. The analysis showed that the observed number of SUD after vaccination were less than the expected number of cases [38]. Another study from Italy assessed the incidence of SUD and its association with the hexavalent vaccine. During the period 2005 - 2009, three deaths were reported within two weeks following the administration of hexavalent vaccines, in approximately 2.5 million vaccinated infants and 7.5 million doses [33].

Hexavalent vaccine co-administered with other paediatric vaccines

In comparison to the administration of the hexavalent vaccine alone, fever seemed to occur more frequently when this was co-administered with other paediatric vaccines $(11.0 \%-57.3 \% \mathrm{vs}$ $30.8-76.7 \%)$. The increased incidence of fever when the hexavalent vaccine was co-administered with other paediatric vaccines is already known and the information is found in the PI of Infanrix ${ }^{\mathrm{TM}}$ hexa as well [11]. uAEs were not reported more frequently in the co-administered groups compared to those who received the hexavalent vaccine alone $(17.7 \%-48.5 \%$ vs $33.7 \%-51.1 \%)$.

Incidence of solicited general symptoms at each dose (\% of subjects)

\begin{tabular}{|c|c|c|c|c|c|c|c|c|c|c|c|c|c|c|c|c|c|}
\hline \multirow{4}{*}{$\begin{array}{c}\text { Study } \\
\text { (Vaccination } \\
\text { schedule in } \\
\text { months) }\end{array}$} & \multirow[t]{4}{*}{ Vaccine } & \multicolumn{16}{|c|}{ General symptoms (overall/Grade $3^{\mathrm{a}}$ ) } \\
\hline & & \multirow{2}{*}{\multicolumn{4}{|c|}{ Fever }} & \multirow{2}{*}{\multicolumn{4}{|c|}{$\begin{array}{c}\text { Fussiness } \\
\text { Dose }\end{array}$}} & \multirow{2}{*}{\multicolumn{4}{|c|}{ Drowsiness }} & \multirow{2}{*}{\multicolumn{4}{|c|}{$\begin{array}{c}\text { Loss of appetite } \\
\text { Dose }\end{array}$}} \\
\hline & & & & & & & & & & & & & & & & & \\
\hline & & 1 & 2 & 3 & 4 & 1 & 2 & 3 & 4 & 1 & 2 & 3 & 4 & 1 & 2 & 3 & 4 \\
\hline \multirow[t]{2}{*}{$\begin{array}{c}\text { Gabutti et al } \\
{[17](3,5,11)}\end{array}$} & $f$ & $\begin{array}{c}24.4 / \\
1.4\end{array}$ & $\begin{array}{c}32.5 / \\
0.5\end{array}$ & $\begin{array}{c}31.1 / \\
1.0\end{array}$ & - & $\begin{array}{c}32.7 / \\
0.9\end{array}$ & $\begin{array}{c}31.1 \\
0.9\end{array}$ & $\begin{array}{c}25.2 \\
1.0\end{array}$ & - & $\begin{array}{c}30.9 / \\
0.9\end{array}$ & $\begin{array}{c}25.0 / \\
0.5\end{array}$ & $\begin{array}{c}21.4 / \\
0.5\end{array}$ & & $\begin{array}{c}20.7 / \\
0.5\end{array}$ & $\begin{array}{c}16.0 / \\
0.5\end{array}$ & 1.0 & \\
\hline & & $\begin{array}{c}18.5 / \\
0.5\end{array}$ & $\begin{array}{c}23.2 / \\
0.0\end{array}$ & \begin{tabular}{|c|}
$28.8 /$ \\
3.4
\end{tabular} & - & $\begin{array}{c}34.71 \\
6.5\end{array}$ & $\begin{array}{c}33.8 \\
2.9\end{array}$ & $\begin{array}{c}25.9 \\
2.4\end{array}$ & - & $\begin{array}{c}29.6 \\
2.3\end{array}$ & $\begin{array}{c}25.6 \\
1.4\end{array}$ & $\begin{array}{c}19.5 / \\
1.0\end{array}$ & - & $\begin{array}{c}21.3 \\
1.9\end{array}$ & $\begin{array}{c}15.0 / \\
1.4\end{array}$ & $\begin{array}{c}18.5 / \\
1.0\end{array}$ & - \\
\hline \multirow{2}{*}{$\begin{array}{c}\text { Tichmann et } \\
\text { al }[30,31](2, \\
4,6,11-23)\end{array}$} & Infan & $\begin{array}{c}15.7 / \\
0.0\end{array}$ & $\begin{array}{c}18.8 / \\
0.0\end{array}$ & $\begin{array}{c}18.7 / \\
0.0\end{array}$ & $\begin{array}{c}31.3 / \\
2.4\end{array}$ & $\begin{array}{c}36.1 / \\
1.7\end{array}$ & $\begin{array}{c}38.8 \\
2.7\end{array}$ & $\begin{array}{c}30.6 \\
1.4\end{array}$ & $\begin{array}{r}38.6 \\
1.8\end{array}$ & $\begin{array}{c}46.5 \\
0.9\end{array}$ & $\begin{array}{c}38.8 / \\
0.9\end{array}$ & $\begin{array}{c}29.2 / \\
0.9\end{array}$ & $\begin{array}{c}39.2 / \\
1.2\end{array}$ & $\begin{array}{c}23.0 \\
0.0\end{array}$ & $\begin{array}{c}20.1 / \\
1.3\end{array}$ & \begin{tabular}{|c|}
$16.9 /$ \\
0.5
\end{tabular} & $\begin{array}{c}30.1 / \\
0.6\end{array}$ \\
\hline & & $9.2 /$ & $\begin{array}{c}10.2 / \\
0.0\end{array}$ & \begin{tabular}{|c|}
$16.8 /$ \\
0.5
\end{tabular} & & \begin{tabular}{|c|}
$39.4 /$ \\
2.8
\end{tabular} & $\begin{array}{c}2.1 \\
38.1 \\
1.9\end{array}$ & & $\begin{array}{r}40.5 \\
0.6\end{array}$ & & $\begin{array}{c}37.2 / \\
1.4\end{array}$ & 32.21 & $\begin{array}{c}1.2 \\
35.6 \\
0.6\end{array}$ & $\begin{array}{c}24.3 \\
0.0\end{array}$ & $\begin{array}{c}21.4 / \\
0.5\end{array}$ & $\begin{array}{c}18.2 / \\
0.9\end{array}$ & 23.30 \\
\hline \multirow[t]{2}{*}{$\begin{array}{c}\text { Kilpi et al }[20] \\
(3,5,11-12)\end{array}$} & & $\begin{array}{c}19.2 / \\
0.0\end{array}$ & $\begin{array}{c}29.1 / \\
0.0\end{array}$ & $\begin{array}{c}35.3 / \\
2.9\end{array}$ & 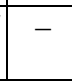 & $\begin{array}{c}58.4 / \\
2.9\end{array}$ & $\begin{array}{c}50.8 / \\
3.3\end{array}$ & & 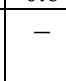 & & $\begin{array}{c}37.7 / \\
0.4\end{array}$ & 0.4 & - & $\begin{array}{c}20.0 / \\
0.8\end{array}$ & $\begin{array}{c}18.4 / \\
0.4\end{array}$ & 2.9 & 0.0 \\
\hline & & \begin{tabular}{|c|}
$28.0 /$ \\
0.0
\end{tabular} & $\begin{array}{c}35.0 / \\
0.0\end{array}$ & \begin{tabular}{|c|}
$42.6 /$ \\
2.1 \\
\end{tabular} & - & $\begin{array}{l}67.5 \\
/ 9.3 \\
\end{array}$ & $\begin{array}{c}56.0 \\
3.3 \\
\end{array}$ & $\begin{array}{c}55.3 \\
4.6 \\
\end{array}$ & - & & $\begin{array}{c}40.7 / \\
0.4 \\
\end{array}$ & \begin{tabular}{|c|}
$45.6 /$ \\
1.7 \\
\end{tabular} & - & $\begin{array}{c}28.0 \\
0.0\end{array}$ & $\begin{array}{c}25.9 / \\
0.0\end{array}$ & $\begin{array}{c}23.8 \\
1.7\end{array}$ & - \\
\hline \multirow{2}{*}{\begin{tabular}{|c|} 
Tichmann et \\
$\boldsymbol{a l}^{\boldsymbol{b}}[32](2,3$ \\
$4,13)$
\end{tabular}} & exa & $\begin{array}{c}24.0 / \\
0.6\end{array}$ & $\begin{array}{c}27.9 / \\
0.0\end{array}$ & & & $\begin{array}{c}32.3 / \\
1.2\end{array}$ & $\begin{array}{c}31.5 \\
0.0\end{array}$ & & & & \begin{tabular}{|c|}
$32.7 /$ \\
0.0
\end{tabular} & $\begin{array}{c}26.4 / \\
0.6\end{array}$ & $\begin{array}{c}31.4 / \\
0.0\end{array}$ & $\begin{array}{c}22.8 \\
0.0\end{array}$ & $\begin{array}{c}18.2 / \\
0.0\end{array}$ & $\begin{array}{c}11.7 \\
0.0\end{array}$ & $\begin{array}{c}24.8 \\
0.0\end{array}$ \\
\hline & $\begin{array}{r}\text { Infar } \\
+1\end{array}$ & $\begin{array}{c}42.8 / \\
1.2\end{array}$ & $\begin{array}{c}49.4 / \\
0.6\end{array}$ & $\begin{array}{c}33.5 / \\
0.0\end{array}$ & $\begin{array}{c}48.6 \\
2.9\end{array}$ & $\begin{array}{c}37.0 \\
2.9\end{array}$ & $\begin{array}{c}36.6 \\
2.9\end{array}$ & $\begin{array}{c}31.8 \\
2.4\end{array}$ & $\begin{array}{r}42.0 \\
1.4 \\
\end{array}$ & $\begin{array}{c}44.5 \\
2.3\end{array}$ & $\begin{array}{c}41.3 / \\
1.2\end{array}$ & $\begin{array}{c}32.4 / \\
1.2\end{array}$ & $\begin{array}{c}44.9 / \\
2.2\end{array}$ & $\begin{array}{c}24.9 \\
0.0\end{array}$ & $\begin{array}{c}27.9 / \\
0.6\end{array}$ & \begin{tabular}{|c|}
$14.7 /$ \\
0.0
\end{tabular} & $\begin{array}{c}34.8 / \\
2.2\end{array}$ \\
\hline \multirow[t]{2}{*}{\begin{tabular}{|c|} 
Vesikari et al \\
{$[37](3,5,11-12)$}
\end{tabular}} & $\begin{array}{l}\text { Infan } \\
+ \text { Pre } \\
\end{array}$ & $\begin{array}{c}26.3 / \\
0.0\end{array}$ & $\begin{array}{c}49.8 / \\
1.5 \\
\end{array}$ & $\begin{array}{c}48.1 / \\
1.5\end{array}$ & - & $\begin{array}{c}76.6 / \\
6.2 \\
\end{array}$ & $\begin{array}{c}74.2 \\
2.2 \\
\end{array}$ & $\begin{array}{c}74.7 \\
1.9\end{array}$ & - & \begin{tabular}{|c}
$58.8 /$ \\
0.7
\end{tabular} & $\begin{array}{c}49.4 \\
2.2\end{array}$ & $\begin{array}{c}52.1 / \\
1.5\end{array}$ & - & $\begin{array}{c}28.5 \\
0.7\end{array}$ & $\mid \begin{array}{c}28.0 \\
0.0\end{array}$ & \begin{tabular}{|c|}
$48.9 /$ \\
0.8
\end{tabular} & - \\
\hline & $\begin{array}{l}\text { 政 } \\
3^{\mathbb{B}} \\
\end{array}$ & $\begin{array}{c}46.3 / \\
0.0 \\
\end{array}$ & $\begin{array}{c}61.7 / \\
0.7\end{array}$ & $\begin{array}{c}52.1 / \\
2.3 \\
\end{array}$ & - & $\begin{array}{c}81.9 / \\
4.8 \\
\end{array}$ & \begin{tabular}{|c|}
76.6 \\
4.1 \\
\end{tabular} & \begin{tabular}{|c|}
75.6 \\
3.4 \\
\end{tabular} & - & $\begin{array}{c}60.5 \\
1.8 \\
\end{array}$ & $\begin{array}{c}49.8 / \\
0.7 \\
\end{array}$ & $\begin{array}{c}53.0 / \\
1.1 \\
\end{array}$ & - & \begin{tabular}{|c|}
35.4 \\
0.7 \\
\end{tabular} & $\begin{array}{c}32.0 \\
1.5\end{array}$ & $\begin{array}{c}44.4 / \\
2.6 \\
\end{array}$ & - \\
\hline \multirow{2}{*}{\begin{tabular}{|c|} 
Van den \\
Bergh $^{c}[35,36]$ \\
$(2,3,4,11-13)$
\end{tabular}} & $\begin{array}{l}\text { Infanr } \\
+ \text { Sy }\end{array}$ & \begin{tabular}{|c}
$33.8 /$ \\
0.0
\end{tabular} & $\begin{array}{c}31.7 / \\
0.8\end{array}$ & $\begin{array}{c}26.9 / \\
0.4\end{array}$ & $\begin{array}{c}39.1 / \\
2.3\end{array}$ & $\begin{array}{c}73.1 / \\
6.9\end{array}$ & $\begin{array}{c}69.9 \\
5.4\end{array}$ & \begin{tabular}{|c|}
53.1 \\
7.3
\end{tabular} & $\begin{array}{c}65.2 \\
4.3 \\
\end{array}$ & \begin{tabular}{|c|}
70.4 \\
1.5 \\
\end{tabular} & $\begin{array}{c}60.6 \\
1.9\end{array}$ & $\begin{array}{c}48.8 / \\
2.7\end{array}$ & $\begin{array}{c}51.2 / \\
2.3\end{array}$ & $\begin{array}{c}33.8 \\
1.2 \\
\end{array}$ & $\mid \begin{array}{c}31.7 / \\
0.4\end{array}$ & $\begin{array}{c}24.2 / \\
0.0\end{array}$ & $\begin{array}{c}36.3 \\
2.0\end{array}$ \\
\hline & & $\begin{array}{c}24.2 / \\
0.0\end{array}$ & $\begin{array}{c}23.1 / \\
0.4\end{array}$ & $\begin{array}{c}19.3 / \\
0.4\end{array}$ & $\begin{array}{c}38.8 \\
2.7\end{array}$ & \begin{tabular}{|c|}
$71.2 /$ \\
6.9
\end{tabular} & $\begin{array}{c}67.7 \\
4.6\end{array}$ & \begin{tabular}{|c}
52.9 \\
5.8
\end{tabular} & $\begin{array}{r}62.4 \\
3.1 \\
\end{array}$ & $\begin{array}{c}65.8 \\
2.7\end{array}$ & \begin{tabular}{|c|}
$54.2 /$ \\
1.9
\end{tabular} & $\begin{array}{c}44.0 / \\
1.2\end{array}$ & \begin{tabular}{|c|}
$45.7 /$ \\
1.9
\end{tabular} & $\begin{array}{c}36.2 \\
0.0\end{array}$ & $\begin{array}{c}28.1 / \\
0.8\end{array}$ & $\begin{array}{c}22.8 / \\
0.8\end{array}$ & $\begin{array}{c}32.2 \\
0.0\end{array}$ \\
\hline
\end{tabular}

${ }^{\mathrm{a}}$ Grade 3 defined: fever, rectal temperature $>39.5^{\circ} \mathrm{C}$; drowsiness, prevents normal activity; fussiness, inconsolable or persistent crying; complete loss of appetite; ${ }^{\mathrm{b}}$, in this study the researcher defined grade 3 fever as rectal temperature $>38.5^{\circ} \mathrm{C}$, but for consistency we presented the $\%$ of subjects with the temperature $>39.5^{\circ} \mathrm{C}$; ${ }^{\circ}$, in these studies the researcher defined grade 3 fever as rectal temperature $>$ $40.0^{\circ} \mathrm{C}$, but for consistency we presented the $\%$ of subjects with the temperature $>39.5^{\circ} \mathrm{C}$ 
Among related SAEs, febrile seizure and urticaria found to be vaccine related are labelled AEs for Infanrix $^{\mathrm{TM}}$ hexa and Prevenar $13^{\circledR}[11,15]$. A review, which included data from post-marketing passive surveillance from GSK database for Infanrix ${ }^{\mathrm{TM}}$ hexa, showed pyrexia as the most frequently reported AE worldwide when Infanrix ${ }^{\mathrm{TM}}$ hexa was coadministered with PCV and the incidence of convulsion and hypotonia was $0.2 \%$ and $0.4 \%$, respectively [3]. In our review, we found that one vaccine related hypotonic-hypo-responsive episode was reported in the included trials. Also, one Kawasaki disease was reported in one child after the co-administration of the hexavalent vaccine with PCV and RV in one RCT included is this review. Kawasaki disease is an AE reported in the PI of RotaTeq ${ }^{\circledR}$. This AE is not listed for the hexavalent vaccine $[11,12]$.

\section{Conclusions}

Reviewed studies showed that hexavalent vaccine was generally well tolerated as primary and booster vaccination. No unlabelled AEs were reported in the RCTs. Only 4 vaccine related SAEs were reported in 6,618 vaccinated children with hexavalent vaccine across RCTs.

\section{Conflict of interest}

The authors declare that they have no conflict of interest.

\section{References}

1. Arístegui J, Dal-Ré R, Díez-Delgado J, Marés J, Casanovas JM, García-Corbeira P, De Frutos E, Van Esso D, Verdaquer J, De la Fluor J, Moraga F, Boceta R, García-Martínez JA, Comparison of the reactogenicity and immunogenicity of a combined diphtheria, tetanus, acellular pertussis, hepatitis B, inactivated polio (DTPa-HBV-IPV) vaccine, mixed with the Haemophilus influenzae type B (Hib). Vaccine, 2003; 21(25-26): 3593-3600.

2. Avdicová M, Prikazský V, Hudecková H, Schuerman L, Willems P, Immunogenicity and reactogenicity of a novel hexavalent DTPa-HBVIPV/Hib vaccine compared to separate concomitant injections of DTPa-IPV/Hib and HBV vaccines, when administered according to a 3,5 and 11 month vaccination schedule. Eur J Pediatr., 2002; 161(11): 581-587.

3. Baldo V, Bonanni P, Castro M, Gabutti G, Franco E, Marchetti F, Prato R, Vitale F, Combined hexavalent diphtheria-tetanus-acellular pertussishepatitis B-inactivated poliovirus-Haemophilus influenzae type $\mathrm{B}$ vaccine; Infanrix ${ }^{\mathrm{TM}}$ hexa: twelve years of experience in Italy. Hum Vaccin Immunother., 2014; 10(1): 129-137.

4. Berner R, Boisnard F, Thomas S, Mwawasi G, Reynolds D, Safety and immunogenicity of fully liquid DTaP5-IPV-Hib pediatric combination vaccine $\left(\right.$ Pediacel $\left.^{\circledR}\right)$ compared to DTaP3-HBVIPV/Hib (Infanrix ${ }^{\circledR}$ Hexa) when coadministered with heptavalent pneumococcal conjugate vaccine (PCV7) as a booster at 11-18 months of age. Vaccine, 2012; 30(35): 5270-5277.

5. Buicu CF, Moldovan I, Cotoi CT, Chiotoroiu AL, Moisi RE, Rafila A, Vaccination in community pharmacies - a global review and the romanian opportunity, Farmacia, 2018; 66(2): 230-236.

6. Deichmann KA, Ferrera G, Tran C, Thomas S, Eymin C, Baudin M, Immunogenicity and safety of a combined measles, mumps, rubella and varicella live vaccine $\left(\right.$ ProQuad $^{\circledR}$ ) administered concomitantly with a booster dose of a hexavalent vaccine in 12-23month-old infants. Vaccine, 2015; 33(20): 23792386.

7. Demicheli V, Rivetti A, Debalini MG, Di Pietrantonj C, Vaccines for measles, mumps and rubella in children. Cochrane database Syst Rev., 2012; 2(2): CD004407.

8. Duclos P, Safety of immunisation and adverse events following vaccination against hepatitis B. Expert Opin Drug Saf., 2003; 2(3): 225-231.

9. EU Clinical Trials Register. www.clinicaltrialsregister.eu.

10. European Medicines Agency (2012). Hexavac: EPAR-Product information. www.ema.europa.eu.

11. European Medicines Agency (2014). Infanrix Hexa: EPAR-Product information. www.ema.europa.eu.

12. European Medicines Agency (2016). RotaTeq: EPARProduct information. www.ema.europa.eu.

13. European Medicines Agency (2017). Hexacima: EPAR-Product information. www.ema.europa.eu.

14. European Medicines Agency (2017). Hexyon: EPAR-Product information. www.ema.europa.eu.

15. European Medicines Agency (2017). Prevenar 13: EPAR-Product information. www.ema.europa.eu.

16. European Medicines Agency (2017). Vaxelis: EPAR-Product information. www.ema.europa.eu.

17. Gabutti G, Zepp F, Schuerman L, Dentico P, Bamfi F, Soncini R, Habernehl P, Knuf M, Crovari P, Cooperative Italian Group for the Study of Combined Vaccines, Evaluation of the immunogenicity and reactogenicity of a DTPa-HBVIPV combination vaccine Co-administered with a Hib conjugate vaccine either as a single injection of a hexavalent combination or as two separate injections at 3,5 and 11 months of age. Scand $J$ Infect Dis., 2004; 36(8): 585-592.

18. GSK Clinical Study Register. www.gsk-clinicalstudy register.com.

19. Halsey NA, Safety of combination vaccines: perception versus reality. Pediatr Infect Dis J., 2001; 20(11Suppl): S40-44.

20. Kilpi TM, Silfverdal SA, Nilsson L, Syrjänen R, Belloni C, Desole M, Triban C, Storsaeter J, Soila $\mathrm{M}$, Jacquet JM, Immunogenicity and reactogenicity of two diphtheria-tetanus-acellular pertussishepatitis B-inactivated polio virus-Haemophilus influenzae type $\mathrm{B}$ vaccines administered at 3,5 and 11-12 months of age. Hum Vaccine, 2009; 5(1): 1825.

21. Larson HJ, de Figueiredo A, Xiahong Z, Schulz WS, Verger P, Johnston IG, Cook AR, Jones NS, 
FARMACIA, 2018, Vol. 66, 5

The state of vaccine confidence 2016: Global insights through a 67-country survey. EBioMedicine, 2016; 12: $295-301$.

22. Liese JG, Stojanov S, Berut F, Minini P, Harzer E, Jow S, Schödel F, Boslego J, Hoffenbach A, Kronwitter A, Belohradsky BH, Munich Vaccine Study Group, Large scale safety study of a liquid hexavalent vaccine (D-T-acP-IPV-PRP $\sim \mathrm{T}-\mathrm{HBs}$ ) administered at 2, 4, 6 and 12-14 months of age. Vaccine, 2001; 20(3-4): 448-454.

23. Macias M, Lanata CF, Zambrano B, Gil AI, Amemiya I, Mispireta M, Ecker L, Santos-Lima E, Safety and immunogenicity of an investigational fully liquid hexavalent DTaP-IPV-Hep B-PRP-T vaccine at two, four and six months of age compared with licensed vaccines in Latin America. Pediatr Infect Dis J., 2012; 31(8): e126-132.

24. Mallet E, Belohradsky BH, Lagos R, Gothefors L, Camier P, Carrière JP, Kanra G, Hoffenbach A, Lanque J, Undereiner F, Roussel F, Reinert P, Flodmark CE, Stojanov S, Liese J, Levine MM, Munoz A, Schödel F, Hessel L, Hexvalent Vaccine Trial Study Group. A liquid hexavalent combined vaccine against diphtheria, tetanus, pertussis, poliomyelitis, Haemophilus influenzae type $\mathrm{B}$ and hepatitis B: review of immunogenicity and safety. Vaccine, 2004; 22(11-12): 1343-1357.

25. Mikaeloff Y, Caridade G, Suissa S, Tardieu M, Hepatitis $B$ vaccine and the risk of CNS inflammatory demyelination in childhood. Neurology, 2009; 72(10): 873-880.

26. Rennels $\mathrm{MB}$, Extensive swelling reactions occurring after booster doses of diphtheria-tetanusacellular pertussis vaccines. Semin Pediatr Infect Dis., 2003; 14(3): 196-198.

27. Saenger R, Maechler G, Potreck M, Zepp F, Knuf M, Habermehl P, Schuerman L, Booster vaccination with hexavalent DTPa-HBV-IPV/Hib vaccine in the second year of life is as safe as concomitant DTPa-IPV/Hib + HBV administered separately. Vaccine, 2005; 23(9): 1135-1143.

28. Salmon DA, Dudley MZ, Glanz JM, Omer SB, Vaccine hesitancy: Causes, consequences, and a call to action. Am J Prev Med., 2015; 49(6): S391-398.

29. Silfverdal SA, Icardi G, Vesikari T, Flores SA, Pagnoni MF, Xu J, Liu GF, Stek JE, Boisnard F, Thomas S, Ziani E, Lee AW, A Phase III randomized, double-blind, clinical trial of an investigational hexa valent vaccine given at 2,4 , and 11-12 months. Vaccine, 2016; 34(33): 3810-3816.

30. Tichmann I, Grunert D, Habash S, Preidel H, Schult $\mathrm{R}$, Pfletschinger U, Gildberg PK, Meurice F, Sänger R, Persistence of antibodies in children primed with two different hexavalent diphtheria, tetanus, acellular pertussis, hepatitis B, inactivated poliovirus and Haemophilus influenzae type B vaccines and evaluation of booster vaccination. Hum Vaccin., 2006; 2(6): 249-254.

31. Tichmann I, Preidel H, Grunert D, Habash S, Schult R, Maier R, Gildberg PK, Sengespeik HC, Meurice F, Comparison of the immunogenicity and reactogenicity of two commercially available hexavalent vaccines administered as a primary vaccination course at 2,4 and 6 months of age. Vaccine, 2005; 23(25): 3272-3279.

32. Tichmann-Schumann I, Soemantri P, Behre U, Disselhoff J, Mahler H, Maechler G, Sänger R, Jacquet JM, Schuerman L, Immunogenicity and reactogenicity of four doses of diphtheria-tetanusthree-component acellular pertussis-hepatitis Binactivated polio virus-Haemophilus influenzae type B vaccine coadministered with 7-valent pneumococcal conjugate vaccine. Pediatr Infect Dis J., 2005; 24(1): 70-77.

33. Traversa G, Spila-Alegiani S, Bianchi C, Ciofi degli Atti M, Frova L, Massari M, Raschetti R, Salmaso S, Scalia Tomba G, Hera Study Group, Sudden unexpected deaths and vaccinations during the first two years of life in Italy: A case series study. PLoS One, 2011; 6(1): 1-10.

34. US National Library of Medicine. www.clinicaltrials.gov.

35. van den Bergh MR, Spijkerman J, François N, Swinnen K, Borys D, Schuerman L, Veenhoven RH, Sanders EA, Immunogenicity, Safety and reactogenicity of a booster dose of the 10-valent pneumococcal nontypeable $H$. influenzae protein $\mathrm{D}$ conjugate vaccine coadministered with DTPa-IPV-Hib in Dutch children: A randomized controlled trial. Pediatr Infect Dis J., 2016; 35(7): e206-219.

36. van den Bergh MR, Spijkerman J, François N, Swinnen K, Borys D, Schuerman L, Veenhoven RH, Sander EA, Immunogenicity, safety, and reactogenicity of the 10-valent pneumococcal nontypeable Haemophilus influenzae protein $\mathrm{D}$ conjugate vaccine and DTPa-IPV-Hib when coadministered as a 3-dose primary vaccination schedule in The Netherlands: A randomized controlled trial. Pediatr Infect Dis J., 2011; 30(9): e170-178.

37. Vesikari T, Silfverdal SA, Jordanov E, Feroldi E, A randomized, controlled study of DTaP-IPV-HB-PRP-T, a fully liquid hexavalent vaccine, administered in a 3-, 5- and 11- to 12-month schedule. Pediatr Infect Dis J., 2017; 36(1): 87-93.

38. von Kries R, Toschke AM, Straßburger K, Kundi M, Kalies H, Nennstiel U, Jorch G, Rosenbauer J, Giani G, Sudden and unexpected deaths after the administration of hexavalent vaccines (diphtheria, tetanus, pertussis, poliomyelitis, hepatitis B, Haemophilius influenzae type B): is there a signal?. Eur J Pediatr., 2005; 164(2): 61-69.

39. Wakefield A, Murch S, Anthony A, Linnell J, Casson D, Malik M, Berelowitz M, Dhillon AP, Thomson MA, Harvey P, Valentine A, Davies SE, WalkerSmith JA, RETRACTED: Ileal-lymphoid-nodular hyperplasia, non-specific colitis, and pervasive developmental disorder in children. Lancet, 1998; 351(9103): 637-641.

40. Wallace AS, Mantel C, Mayers G, Mansoor O, Gindler JS, Hyde TB, Experiences with provider and parental attitudes and practices regarding the administration of multiple injections during infant vaccination visits: Lessons for vaccine introduction. Vaccine, 2014; 32(41): 5301-5310.

41. World Health Organization (2011). European countries must take action now to prevent continued measles outbreaks in 2012. www.euro.who.int. 
FARMACIA, 2018, Vol. 66, 5

42. World Health Organization (2016). Immunization coverage. www.who.int.

43. World Health Organization (2017). WHO EpiData. www.euro.who.int.

44. World Health Organization. Hepatitis B vaccines: WHO position paper. Wkly Epidemiol Rec., 2009; 84(40): 405-420.

45. Zepp F, Behre U, Kindler K, Laakmann KH, Pankow-Culot H, Mannhardt-Laakmann W, Beckers F,
Descamps D, Immunogenicity and safety of a tetravalent measles-mumps-rubella-varicella vaccine co-administered with a booster dose of a combined diphtheria-tetanus- acellular pertussis-hepatitis Binactivated poliovirus-Haemophilus influenzae type B conjugate vaccine. Eur J Pediatr., 2007; 166(8): 857-864. 